\title{
On extremal mappings in complex ellipsoids
}

\author{
by Armen Edigarian (Kraków)
}

\begin{abstract}
Using a generalization of [Pol] we present a description of complex geodesics in arbitrary complex ellipsoids.
\end{abstract}

1. Introduction and the main results. Let $\mathcal{E}(p):=\left\{\left|z_{1}\right|^{2 p_{1}}+\ldots+\right.$ $\left.\left|z_{n}\right|^{2 p_{n}}<1\right\} \subset \mathbb{C}^{n}$, where $p=\left(p_{1}, \ldots, p_{n}\right), p_{j}>0, j=1, \ldots, n ; \mathcal{E}(p)$ is called a complex ellipsoid.

The aim of the paper is to characterize complex $\varkappa_{\mathcal{E}(p)^{-}}$and $\widetilde{k}_{\mathcal{E}(p)^{- \text {geode- }}}$ sics. The case where $\mathcal{E}(p)$ is convex (i.e. $p_{1}, \ldots, p_{n} \geq 1 / 2$ ) has been solved in [Jar-Pfl-Zei]. The paper is inspired by methods of [Pol].

Let $D \subset \mathbb{C}^{n}$ be a domain and let $\varphi \in \mathcal{O}(E, D)$, where $E$ denotes the unit disk in $\mathbb{C}$ and $\mathcal{O}(\Omega, D)$ is the set of all holomorphic mappings $\Omega \rightarrow D$. Recall that $\varphi$ is said to be a $\varkappa_{D}$-geodesic if there exists $(z, X) \in D \times \mathbb{C}^{n}$ such that:

- $\varphi(0)=z$ and $\varphi^{\prime}(0)=\lambda_{\varphi} X$ for some $\lambda_{\varphi}>0$,

- for any $\psi \in \mathcal{O}(E, D)$ such that $\psi(0)=z$ and $\psi^{\prime}(0)=\lambda_{\psi} X$ with $\lambda_{\psi}>0$, we have $\lambda_{\psi} \leq \lambda_{\varphi}$.

We say that $\varphi$ is a $\widetilde{k}_{D}$-geodesic if there exists $(z, w) \in D \times D$ such that:

- $\varphi(0)=z$ and $\varphi\left(\sigma_{\varphi}\right)=w$ for some $\sigma_{\varphi} \in(0,1)$,

- for any $\psi \in \mathcal{O}(E, D)$ such that $\psi(0)=z$ and $\psi\left(\sigma_{\psi}\right)=w$ with $\sigma_{\psi}>0$, we have $\sigma_{\varphi} \leq \sigma_{\psi}$; cf. [Pan].

Let us fix some further notations: $\mathbb{C}^{n} ;$

- $H^{\infty}\left(\Omega, \mathbb{C}^{n}\right):=$ the space of all bounded holomorphic mappings $\Omega \rightarrow$

- $\|f\|_{\infty}:=\sup \{\|f(z)\|: z \in \Omega\}, f \in H^{\infty}\left(\Omega, \mathbb{C}^{n}\right)$, where \|\| denotes the Euclidean norm in $\mathbb{C}^{n}$;

1991 Mathematics Subject Classification: Primary 32H15.

Key words and phrases: complex ellipsoid, geodesics, extremal mapping. 
- $f^{*}(\zeta):=$ the non-tangential boundary value of $f$ at $\zeta \in \partial E, f \in$ $H^{\infty}\left(E, \mathbb{C}^{n}\right)$

- $\mathcal{A}\left(\Omega, \mathbb{C}^{n}\right):=\mathcal{C}\left(\bar{\Omega}, \mathbb{C}^{n}\right) \cap \mathcal{O}\left(\Omega, \mathbb{C}^{n}\right)$

- $z \cdot w:=\left(z_{1} w_{1}, \ldots, z_{n} w_{n}\right), z \bullet w:=\sum_{j=1}^{n} z_{j} w_{j}, z=\left(z_{1}, \ldots, z_{n}\right), w=$ $\left(w_{1}, \ldots, w_{n}\right) \in \mathbb{C}^{n}$

- $A_{\nu}:=\{z \in \mathbb{C}: \nu<|z|<1\}, \nu \in(0,1)$

- $\operatorname{PSH}(\Omega):=$ the set of all plurisubharmonic functions on $\Omega$.

Fix $w_{1}, \ldots, w_{N} \in \mathcal{A}\left(A_{\nu}, \mathbb{C}^{n}\right)$ and define

$\Phi_{j}(h)=\frac{1}{2 \pi} \int_{0}^{2 \pi} \operatorname{Re}\left(h^{*}\left(e^{i \theta}\right) \bullet w_{j}\left(e^{i \theta}\right)\right) d \theta, \quad h \in H^{\infty}\left(E, \mathbb{C}^{n}\right), j=1, \ldots, N$.

We say that the functionals $\Phi_{1}, \ldots, \Phi_{N}$ are linearly independent if for arbitrary $s=\left(s_{1}, \ldots, s_{n}\right), g \in H^{\infty}\left(E, \mathbb{C}^{n}\right)$, and $\lambda_{1}, \ldots, \lambda_{N} \in \mathbb{R}$ such that $s_{k}$ nowhere vanishes on $E, k=1, \ldots, n$, and $g(0)=0$ the following implication is true: if $\sum_{j=1}^{N} \lambda_{j} w_{j} \cdot s^{*}=g^{*}$ on a subset of $\partial E$ of positive measure, then $\lambda_{1}=\ldots=\lambda_{N}=0$.

Later on, we always assume that the functionals $\Phi_{1}, \ldots, \Phi_{N}$ are linearly independent.

$\operatorname{Problem}(\mathcal{P})$. Given a bounded domain $D \subset \mathbb{C}^{n}$ and numbers $a_{1}, \ldots$ $\ldots, a_{N} \in \mathbb{R}$, find a mapping $f \in \mathcal{O}(E, D)$ such that $\Phi_{j}(f)=a_{j}, j=$ $1, \ldots, N$, and there is no mapping $g \in \mathcal{O}(E, D)$ with

$$
\Phi_{j}(g)=a_{j}, \quad j=1, \ldots, N, \quad g(E) \Subset D .
$$

Any solution of $(\mathcal{P})$ is called an extremal mapping for $(\mathcal{P})$ or, simply, an extremal.

Problem $(\mathcal{P})$ is a generalization of Problem $(\mathrm{P})$ from $[\mathrm{Pol}]$.

We say that problem $(\mathcal{P})$ is of $m$-type if there exists a polynomial $Q(\zeta)=$ $\prod_{k=1}^{m}\left(\zeta-\sigma_{k}\right)$ with $\sigma_{1}, \ldots, \sigma_{m} \in E$ such that $Q w_{j}$ extends to a mapping of class $\mathcal{A}\left(E, \mathbb{C}^{n}\right), j=1, \ldots, N$.

One can prove that (for bounded domains $D \subset \mathbb{C}^{n}$ ) any complex $\varkappa_{D^{-}}$ or $\widetilde{k}_{D}$-geodesic may be characterized as an extremal for a suitable problem $(\mathcal{P})$ of 1-type (cf. $\S 4)$

The main result of the paper is the following

TheOrem 1. Let $D \Subset G \Subset \mathbb{C}^{n}$ be domains and let $u \in P S H(G) \cap \mathcal{C}(G)$ be such that $D=\{u<0\}, \partial D=\{u=0\}$. Suppose that $f \in \mathcal{O}(E, D)$ is an extremal for $(\mathcal{P})$. Assume that there exist a set $S \subset \partial E$, a mapping $s=\left(s_{1}, \ldots, s_{n}\right) \in H^{\infty}\left(E, \mathbb{C}^{n}\right)$, a number $\varepsilon>0$, and a function $v: S \times$ $\mathcal{A}\left(E, \mathbb{C}^{n}\right) \rightarrow \mathbb{C}$ such that:

(a) $\partial E \backslash S$ has zero measure,

(b) $f^{*}(\zeta), \nabla u\left(f^{*}(\zeta)\right)$ and $s^{*}(\zeta)$ are defined for all $\zeta \in S$, 
(c) $s_{k}$ nowhere vanishes on $E$ for $k=1, \ldots, n$,

(d) $u\left(f^{*}(\zeta)+s^{*}(\zeta) \cdot h(\zeta)\right)=u\left(f^{*}(\zeta)\right)+2 \operatorname{Re}\left(\nabla u\left(f^{*}(\zeta)\right) \bullet\left(s^{*}(\zeta) \cdot h(\zeta)\right)\right)+$ $v(\zeta, h), \zeta \in S, h \in \mathcal{A}\left(E, \mathbb{C}^{n}\right),\|h\|_{\infty} \leq \varepsilon$,

(e) $\lim _{h \rightarrow 0} \sup \{|v(\zeta, h)|: \zeta \in S\} /\|h\|_{\infty}=0$.

Then

$$
f^{*}(\zeta) \in \partial D \quad \text { for a.a. } \zeta \in \partial E
$$

and there exist $\varrho \in L^{\infty}(\partial E), \varrho>0, g \in H^{\infty}\left(E, \mathbb{C}^{n}\right)$, and $\left(\lambda_{1}, \ldots, \lambda_{N}\right) \in$ $\mathbb{R}^{N} \backslash\{0\}$ such that

$$
\sum_{k=1}^{N} \lambda_{k} w_{k}(\zeta) \cdot s^{*}(\zeta)+g^{*}(\zeta)=\varrho(\zeta) s^{*}(\zeta) \cdot \nabla u\left(f^{*}(\zeta)\right) \quad \text { for a.a. } \zeta \in \partial E .
$$

Remark 2. Under the assumptions of Theorem 1, if $u \in \mathcal{C}^{1}(G) \cap$ $\operatorname{PSH}(G)$, then one can take $s: \equiv(1, \ldots, 1)$.

As an easy corollary to Theorem 1 we obtain

CoRollary 3. Under the assumptions of Theorem 1 , if problem $(\mathcal{P})$ is of $m$-type, then there exist $\varrho \in L^{\infty}(\partial E), \varrho>0$, and $g \in H^{\infty}\left(E, \mathbb{C}^{n}\right)$ such that

$$
g^{*}(\zeta)=Q(\zeta) \varrho(\zeta) s^{*}(\zeta) \cdot \nabla u\left(f^{*}(\zeta)\right) \quad \text { for a.a. } \zeta \in \partial E .
$$

Theorem 1 generalizes Theorems 2 and 3 of [Pol] (cf. Remark 2). The proof of Theorem 1 will be presented in $\S 2$.

Corollary 3 gives a tool for describing the extremal mappings for problems $(\mathcal{P})$ of $m$-type in the case where $D$ is an arbitrary complex ellipsoid $\mathcal{E}(p)$.

Theorem 4. Let $\varphi: E \rightarrow \mathcal{E}(p)$ be an extremal for problem $(\mathcal{P})$ of $m$-type such that $\varphi_{j} \not \equiv 0, j=1, \ldots, n$. Then

$$
\varphi_{j}(\lambda)=a_{j} \prod_{k=1}^{m}\left(\frac{\lambda-\alpha_{k j}}{1-\bar{\alpha}_{k j} \lambda}\right)^{r_{k j}}\left(\frac{1-\bar{\alpha}_{k j} \lambda}{1-\bar{\alpha}_{k 0} \lambda}\right)^{1 / p_{j}}, \quad j=1, \ldots, n,
$$

where

- $a_{1}, \ldots, a_{n} \in \mathbb{C} \backslash\{0\}$,

- $\alpha_{k j} \in \bar{E}, k=1, \ldots, m, j=0, \ldots, n$,

- $r_{k j} \in\{0,1\}$ and, if $r_{k j}=1$, then $\alpha_{k j} \in E$,

- $\sum_{j=1}^{n}\left|a_{j}\right|^{2 p_{j}} \prod_{k=1}^{m}\left(\zeta-\alpha_{k j}\right)\left(1-\bar{\alpha}_{k j} \zeta\right)=\prod_{k=1}^{m}\left(\zeta-\alpha_{k 0}\right)\left(1-\bar{\alpha}_{k 0} \zeta\right), \zeta \in E$.

In particular, if $\varphi$ is a complex $\varkappa_{\mathcal{E}(p)}-$ or $\widetilde{k}_{\mathcal{E}(p)}$-geodesic, then $\varphi$ is of the above form with $m=1$.

Theorem 4 generalizes $\S 6$ of [Pol] and Theorem 1 of [Jar-Pfl-Zei]. The proof of Theorem 4 will be given in $\S \S 3,4$. 
R e m a r $\mathrm{k}$ 5. In the case where $\mathcal{E}(p)$ is convex any mapping described in Theorem 4 with $m=1$ is a complex geodesic in $\mathcal{E}(p)$ ([Jar-Pfl-Zei]). This is no longer true if $\mathcal{E}(p)$ is not convex (cf. [Pfl-Zwo] for the case $n=2, p_{1}=1$, $\left.p_{2}<1 / 2\right)$.

2. Proof of Theorem 1. Note that there are two possibilities: either $u \circ f^{*}=0$ a.e. on $\partial E$ or there exists $\tau>0$ such that the set $\left\{\theta: u\left(f^{*}\left(e^{i \theta}\right)\right)<\right.$ $-\tau\}$ has positive measure. If such a $\tau$ exists, fix one of them. We put

$$
P_{0}:= \begin{cases}\emptyset & \text { in the first case, } \\ \left\{\theta: u\left(f^{*}\left(e^{i \theta}\right)\right)<-\tau\right\} & \text { in the second case }\end{cases}
$$

$A_{0}:=[0,2 \pi) \backslash P_{0}$, and

$p_{s}(h):=\frac{1}{2 \pi} \int_{A_{0}}\left[\operatorname{Re}\left(s^{*}\left(e^{i \theta}\right) \cdot \nabla u\left(f^{*}\left(e^{i \theta}\right)\right) \bullet h\left(e^{i \theta}\right)\right)\right]^{+} d \theta \quad$ for $h \in L^{1}\left(\partial E, \mathbb{C}^{n}\right)$,

where $L^{1}\left(\partial E, \mathbb{C}^{n}\right)$ denotes the space of all Lebesgue integrable mappings $\partial E \rightarrow \mathbb{C}^{n}$.

Remark 6. (a) Under the assumptions of Theorem 1, there exists $M>0$ such that

$$
\left\|s^{*}(\zeta) \cdot \nabla u\left(f^{*}(\zeta)\right)\right\| \leq M \quad \text { for a.a. } \zeta \in \partial E .
$$

(b) $p_{s}(h)$ is a seminorm on $H^{1}\left(E, \mathbb{C}^{n}\right)$ and $p_{s}(h) \leq M\|h\|_{1}$, where $H^{1}(E)$ denotes the first Hardy space of holomorphic functions,

$$
H^{1}\left(E, \mathbb{C}^{n}\right):=\left\{\left(f_{1}, \ldots, f_{n}\right): f_{j} \in H^{1}(E)\right\},
$$

and \|\|$_{1}$ denotes the norm in $H^{1}\left(E, \mathbb{C}^{n}\right)$.

The proof of Theorem 1 is based on the following result.

Lemma 7 (cf. [Pol], Lemma 6). Under the assumptions of Theorem 1 there exist $T>0, j \in\{1, \ldots, N\}$, and $\delta \in\{-1,1\}$ such that

$$
\delta \Phi_{j}(s \cdot h) \leq T p_{s}(h)
$$

for $h \in X_{j}:=\left\{h \in H^{1}\left(E, \mathbb{C}^{n}\right): \Phi_{l}(s \cdot h)=0, l \neq j\right\}$.

Let us for a while assume that we already have Lemma 7.

Proof of Theorem 1. By Lemma 7 there exist $T>0, \delta \in\{-1,1\}$, and $j \in\{1, \ldots, N\}$ such that

$$
\delta \Phi_{j}(s \cdot h) \leq T p_{s}(h) \quad \text { for } h \in X_{j} .
$$

Let $\widetilde{\Phi}(h):=\delta \Phi_{j}(s \cdot h), h \in X_{j}$. Using the Hahn-Banach theorem we can extend $\widetilde{\Phi}$ to $L^{1}\left(\partial E, \mathbb{C}^{n}\right)$ (we denote this extension by $\Phi$ ) in such a way that

$$
\Phi(h) \leq T p_{s}(h) \quad \text { for } h \in L^{1}\left(\partial E, \mathbb{C}^{n}\right) .
$$


We know that $p_{s}(h) \leq M|h|_{1}$, where $|h|_{1}$ denotes the norm in $L^{1}\left(E, \mathbb{C}^{n}\right)$. So $\Phi$ is continuous on $L^{1}\left(\partial E, \mathbb{C}^{n}\right)$. By Riesz's theorem, $\Phi$ can be represented as

$$
\Phi(h)=\frac{1}{2 \pi} \int_{0}^{2 \pi} \operatorname{Re}\left(h^{*}\left(e^{i \theta}\right) \bullet \widetilde{w}\left(e^{i \theta}\right)\right) d \theta, \quad \text { where } \widetilde{w} \in L^{\infty}\left(\partial E, \mathbb{C}^{n}\right) .
$$

It is easy to see that there are $\lambda_{1}, \ldots, \lambda_{N}$, not all zero, such that $\Phi(h)=$ $\sum_{k=1}^{N} \lambda_{k} \Phi_{k}(s \cdot h)$ for $h \in H^{1}\left(E, \mathbb{C}^{n}\right)$. We denote by $G$ the linear functional on $L^{1}\left(\partial E, \mathbb{C}^{n}\right)$ defined by the formula

$$
G(h):=\frac{1}{2 \pi} \int_{0}^{2 \pi} \operatorname{Re}\left(\sum_{k=1}^{N} \lambda_{k} w_{k}\left(e^{i \theta}\right) \bullet s^{*}\left(e^{i \theta}\right) \cdot h\left(e^{i \theta}\right)\right) d \theta .
$$

Then $\Phi(h)-G(h)=0$ for $h \in H^{1}\left(E, \mathbb{C}^{n}\right)$. By the theorem of F. \& M. Riesz it follows that there exists $g \in H^{\infty}\left(E, \mathbb{C}^{n}\right), g(0)=0$, such that

$$
\widetilde{w}-s^{*} \cdot \sum_{k=1}^{N} \lambda_{k} w_{k}=g^{*}
$$

We have

$$
\text { (1) } \begin{aligned}
\Phi(h) & =\frac{1}{2 \pi} \int_{0}^{2 \pi} \operatorname{Re}\left[\left(\sum_{k=1}^{N} \lambda_{k} w_{k}\left(e^{i \theta}\right) \cdot s^{*}\left(e^{i \theta}\right)+g^{*}\left(e^{i \theta}\right)\right) \bullet h^{*}\left(e^{i \theta}\right)\right] d \theta \\
& \leq T \frac{1}{2 \pi} \int_{A_{0}}\left[\operatorname{Re}\left(s^{*}\left(e^{i \theta}\right) \cdot \nabla u\left(f^{*}\left(e^{i \theta}\right)\right) \bullet h^{*}\left(e^{i \theta}\right)\right)\right]^{+} d \theta
\end{aligned}
$$

for any $h \in H^{1}\left(E, \mathbb{C}^{n}\right)$. We see that the right-hand side is zero for any $h \in H^{1}\left(E, \mathbb{C}^{n}\right)$ (hence, for any $\left.h \in L^{1}\left(\partial E, \mathbb{C}^{n}\right)\right)$ such that

$$
\operatorname{Re}\left(s^{*}\left(e^{i \theta}\right) \cdot \nabla u\left(f^{*}\left(e^{i \theta}\right)\right) \bullet h^{*}\left(e^{i \theta}\right)\right) \leq 0
$$

on $\partial E \backslash\left(P_{0} \cup\left\{\zeta \in \partial E: s^{*}(\zeta) \cdot \nabla u\left(f^{*}(\zeta)\right)=0\right\}\right)$.

Hence

$$
\sum_{k=1}^{N} \lambda_{k} w_{k} \cdot s^{*}+g^{*}=0 \quad \text { a.e. on } P_{0} \cup\left\{\zeta \in \partial E: s^{*}(\zeta) \cdot \nabla u\left(f^{*}(\zeta)\right)=0\right\} \text {. }
$$

We know that $\Phi_{1}, \ldots, \Phi_{N}$ are linearly independent, so the Lebesgue measures of $P_{0}$ and of $\left\{\zeta \in \partial E: s^{*}(\zeta) \cdot \nabla u\left(f^{*}(\zeta)\right)=0\right\}$ are zero. Hence

$$
\sum_{k=1}^{N} \lambda_{k} w_{k}(\zeta) \cdot s^{*}(\zeta)+g^{*}(\zeta)=\varrho(\zeta) s^{*}(\zeta) \cdot \nabla u\left(f^{*}(\zeta)\right),
$$

where $\varrho(\zeta) \in \mathbb{C} \backslash\{0\}$ for a.a. $\zeta \in \partial E$. Now, it is enough to remark that condition (1) implies that $0<\varrho \leq T$ a.e. on $\partial E$.

Now, we are going to prove Lemma 7. 
Proof of Lemma 7. Suppose that the lemma is not true. Then for each $j \in\{1, \ldots, N\}$ and $m \in \mathbb{N}$ there are $h_{j m}^{+}, h_{j m}^{-} \in X_{j}$ such that

$$
\Phi_{j}\left(s \cdot h_{j m}^{+}\right)>m p_{s}\left(h_{j m}^{+}\right), \quad-\Phi_{j}\left(s \cdot h_{j m}^{-}\right)>m p_{s}\left(h_{j m}^{-}\right) .
$$

We may assume that $h_{j m}^{+}, h_{j m}^{-} \in \mathcal{A}\left(E, \mathbb{C}^{n}\right)$ and that

$$
\Phi_{j}\left(s \cdot h_{j m}^{+}\right)=1, \quad \Phi_{j}\left(s \cdot h_{j m}^{-}\right)=-1 .
$$

For any $q=\left(q_{1}^{+}, q_{1}^{-}, \ldots, q_{N}^{+}, q_{N}^{-}\right) \in \mathbb{R}_{+}^{2 N}$ we define the function

$$
f_{q m}=f+\sum_{j=1}^{N}\left(q_{j}^{+} s \cdot h_{j m}^{+}+q_{j}^{-} s \cdot h_{j m}^{-}\right)=f+s \cdot h_{q m}
$$

and the linear mapping $A: \mathbb{R}_{+}^{2 N} \rightarrow \mathbb{R}^{N}, A(q):=\left(q_{1}^{+}-q_{1}^{-}, \ldots, q_{N}^{+}-q_{N}^{-}\right)$. Note that $\Phi_{j}\left(f_{q m}\right)-\Phi_{j}(f)=A(q)_{j}$.

LEMma 8 (see [Pol], Lemma 7). Let $u$ be a non-positive subharmonic function in $E$ and let $\triangle u$ be the Riesz measure of $u$. Suppose that one of the following conditions is true:

(a) $\triangle u\left(r_{0} E\right)>a>0$ for some $r_{0} \in(0,1)$,

(b) for some set $Z \subset[0,2 \pi)$ with positive measure, the upper radial limit of $u$ at $\zeta \in Z$ does not exceed $-a<0$ (i.e. $\lim _{\sup _{r \rightarrow 1}} u(r \zeta) \leq-a$ ).

Then $u(\zeta) \leq-C(1-|\zeta|)$, where $C>0$ is a constant depending only on $r_{0}, a$, and $Z$.

Let $u_{0}:=u \circ f$.

Lemma 9. There exist a constant $C>0$ and constants $t_{m}>0, m \in \mathbb{N}$, such that for $\|q\|<t_{m}$ we have

(a) $f_{q m} \in \mathcal{O}(E, G)$ (so, we define $\left.u_{q m}:=u \circ f_{q m}\right)$,

(b) $u_{q m}(\zeta) \leq v_{q m}(\zeta):=C \ln |\zeta|+\frac{1}{2 \pi} \int_{A_{0}}\left[u_{q m}^{*}\left(e^{i \theta}\right)\right]^{+} P(\zeta, \theta) d \theta$ for $|\zeta|>1 / 2$.

Proof. (a) follows from the assumption that $D \Subset G$.

(b) Suppose that there exists $r_{0} \in(0,1)$ such that $\triangle u_{0}\left(r_{0} E\right)>a>0$. The continuity of $u$ implies that for

$$
\widetilde{u}_{q m}(\zeta):=u_{q m}(\zeta)-\frac{1}{2 \pi} \int_{A_{0}}\left[u_{q m}^{*}\left(e^{i \theta}\right)\right]^{+} P(\zeta, \theta) d \theta, \quad \zeta \in E,
$$

if $t_{m}$ is small enough then $\Delta \widetilde{u}_{q m}(r E)>a / 2$. Hence, from Lemma 8 we get the required result.

If $\triangle u_{0}(r E)=0$ for any $r \in(0,1)$ and $u_{0}^{*}(\zeta)=0$ for a.a. $\zeta \in \partial E$, then by the Riesz representation theorem ([Hay-Ken], Ch. 3.5) we see that $u_{0}$ is harmonic in $E$. But this is a contradiction, since $u_{0} \not \equiv 0$. Hence, $P_{0}$ has 
positive measure. From the continuity of $u$ we conclude that if $t_{m}$ are small enough, then $\left\{\zeta: \widetilde{u}_{q m}(\zeta)<-\tau / 2\right\}$ has positive measure. By Lemma 8 we get the required result.

Let us introduce some new notation: $E_{q m}:=\left\{\zeta \in E: v_{q m}(\zeta)<0\right\}$ and

$$
g_{q m}(\zeta):=\zeta \exp \left\{\frac{1}{2 \pi C} \int_{A_{0}}\left[u_{q m}^{*}\left(e^{i \theta}\right)\right]^{+} S(\zeta, \theta) d \theta\right\} .
$$

Here $S(\zeta, \theta):=\left(\zeta+e^{i \theta}\right) /\left(\zeta-e^{i \theta}\right)$ is the Schwarz kernel.

Remark 10. Note that $C \ln \left|g_{q m}\right|=v_{g m}, v_{q m}(\zeta) \geq C \ln |\zeta|$ (hence, $\left.\left|g_{q m}(\zeta)\right| \geq|\zeta|\right)$, and $E_{q m}=g_{q m}^{-1}(E)$.

Lemma 11 (cf. [Pol], Statement 2). (a) $E_{q m}$ is connected, $0 \in E_{q m}$, (b) $g_{q m}$ maps $E_{q m}$ conformally onto $E$.

Proof. (a) Note that $E_{q m}=\bigcup_{\delta>0}\left\{\zeta: v_{q m}(\zeta)<-\delta\right\}$ and

$$
\left\{\zeta: v_{q m}(\zeta)<-\delta\right\} \subset\left\{\zeta:|\zeta|<e^{-\delta / C}\right\} .
$$

Since $v_{q m}$ is harmonic outside 0 and $v_{q m}^{*}\left(e^{i \theta}\right) \geq 0$, any connected component of $\left\{\zeta: v_{q m}(\zeta)<-\delta\right\}$ must contain 0 .

(b) First let us see that $g_{q m}: E_{q m} \rightarrow E$ is proper. Let $\zeta_{k} \rightarrow \zeta_{0} \in \partial E_{q m}$. If $\zeta_{0} \in \partial E$, then $\left|g_{q m}\left(\zeta_{k}\right)\right| \rightarrow 1$ (since $\left.\left|g_{q m}\right| \geq|\zeta|\right)$. If $\zeta_{0} \in E$, then $\left|g_{q m}\left(\zeta_{k}\right)\right| \rightarrow$ $\left|g_{q m}\left(\zeta_{0}\right)\right|=1$.

Since $g_{q m}^{\prime}(0) \neq 0$ and $g_{q m}^{-1}(0)=\{0\}, g_{q m}$ is conformal.

We define $\widetilde{f}_{q m}(\zeta)=f_{q m}\left(g_{q m}^{-1}(\zeta)\right), \widehat{f}_{q m}(\zeta)=\widetilde{f}_{q m}\left(e^{-\|q\| / m} \zeta\right)$,

and

$$
\widetilde{A}_{m}(q)=\left(\Phi_{1}\left(\widetilde{f}_{q m}\right)-\Phi_{1}(f), \ldots, \Phi_{N}\left(\widetilde{f}_{q m}\right)-\Phi_{N}(f)\right),
$$

$$
\widehat{A}_{m}(q)=\left(\Phi_{1}\left(\widehat{f}_{q m}\right)-\Phi_{1}(f), \ldots, \Phi_{N}\left(\widehat{f}_{q m}\right)-\Phi_{N}(f)\right) .
$$

Remark 12. It is easy to see that $\widetilde{f}_{q m}(E) \subset D, \widehat{f}_{q m}(E) \Subset D$, and $\widetilde{A}_{m}(0)=\widehat{A}_{m}(0)=0$.

The following result explains why we have used functionals of the special form.

Lemma 13. Suppose that

$$
\Phi(h)=\frac{1}{2 \pi} \int_{0}^{2 \pi} \operatorname{Re}\left(h^{*}\left(e^{i \theta}\right) \bullet w\left(e^{i \theta}\right)\right) d \theta,
$$

where $w \in \mathcal{A}\left(A_{\nu}, \mathbb{C}^{n}\right)$ for some $\nu \in(0,1), f \in H^{\infty}\left(E, \mathbb{C}^{n}\right)$, and that $g \in$ $\mathcal{O}(E, E), g(0)=0$. Then

$$
|\Phi(f \circ g)-\Phi(f)| \leq K\|f\|_{\infty} \sup _{\zeta \in E}|g(\nu \zeta)-\nu \zeta|,
$$

where $K>0$ depends only on $\Phi$. 
Proof. We have

$$
\Phi(h)=\frac{1}{2 \pi} \int_{0}^{2 \pi} \operatorname{Re}\left(h\left(\nu e^{i \theta}\right) \bullet w\left(\nu e^{i \theta}\right)\right) d \theta .
$$

Hence

$$
|\Phi(h)| \leq\left(\max _{\zeta \in \partial E}\|w(\nu \zeta)\|\right)\left(\max _{\zeta \in \partial E}\|h(\nu \zeta)\|\right)
$$

But

$$
\|f(g(\nu \zeta))-f(\nu \zeta)\| \leq\left(\sup _{\xi \in E}\left|f^{\prime}(\nu \xi)\right|\right)|g(\nu \zeta)-\nu \zeta|,
$$

and $\sup _{\xi \in E}\left|f^{\prime}(\nu \xi)\right| \leq\|f\|_{\infty} /\left(1-\nu^{2}\right)$

Lemma 14 (cf. [Pol], Statement 3). The mappings $\widetilde{A}_{m}, \widehat{A}_{m}$ are continuous in $q$ for $\|q\|<t_{m}$.

Proof. It is enough to remark that if $q_{k} \rightarrow q$, then $u_{q_{k} m}^{*} \rightarrow u_{q m}^{*}$ uniformly on $\partial E$. Hence $g_{q_{k} m} \rightarrow g_{q m}$ uniformly on compact subsets of $E$. It is evident from the last assertion that also $g_{q_{k} m}^{-1} \rightarrow g_{q m}^{-1}, \widetilde{f}_{q_{k} m} \rightarrow \widetilde{f}_{q m}$, and $\widehat{f}_{q k} \rightarrow \widehat{f}_{q m}$ uniformly on compact sets. Since the $\Phi_{j}$ are continuous with respect to this convergence (this follows easily from (2)), we conclude the proof.

LEMMA 15. For each $b>0$ there is $m_{0} \in \mathbb{N}$ such that for any $m \geq m_{0}$ there is $q_{m}>0$ such that $\left\|A(q)-\widetilde{A}_{m}(q)\right\| \leq b\|q\|$ whenever $\|q\| \leq q_{m}$.

P r o o f. It follows from the definition of $A, \widetilde{A}_{m}$ that it is enough to prove the inequality

$$
\left|\Phi\left(\tilde{f}_{q m}\right)-\Phi\left(f_{q m}\right)\right| \leq b\|q\|
$$

for small $q$, where $\Phi$ is a functional of our special form. By Lemma 14 it is enough to show that

$$
\sup _{\zeta \in \nu E}\left|g_{q m}^{-1}(\zeta)-\zeta\right| \leq b\|q\|
$$

for small $q$. Note that

$$
\sup _{\zeta \in \nu E}\left|g_{q m}^{-1}(\zeta)-\zeta\right| \leq \sup _{\zeta \in \nu E}\left|g_{q m}(\zeta)-\zeta\right|
$$

and for small $q_{m}$ (such that $\left.\left|1-\exp q_{m}\right| \leq 2 q_{m}\right)$ and $\|q\| \leq q_{m}$,

$$
\begin{aligned}
& \left|1-\exp \left(\frac{1}{2 \pi C} \int_{A_{0}}\left[u_{q m}^{*}\left(e^{i \theta}\right)\right]^{+} S(\zeta, \theta) d \theta\right)\right| \\
& \leq 2 \frac{1+\nu}{1-\nu}\left(\frac{1}{2 \pi C} \int_{A_{0}}\left[u_{q m}^{*}\left(e^{i \theta}\right)\right]^{+} d \theta\right)
\end{aligned}
$$


for $\zeta \in \nu E$. Hence, it is enough to show that

$$
\begin{aligned}
\int_{A_{0}}\left[u_{q m}^{*}\left(e^{i \theta}\right)\right]^{+} d \theta \leq & \int_{A_{0}} 2\left[\operatorname{Re}\left(\nabla u\left(f^{*}\left(e^{i \theta}\right)\right) \bullet s^{*}\left(e^{i \theta}\right) \cdot h_{q m}\left(e^{i \theta}\right)\right)\right]^{+} d \theta \\
& +o\left(\left\|h_{q m}\right\|_{\infty}\right) .
\end{aligned}
$$

But $p_{s}\left(h_{q m}\right) \leq\|q\| \max \left\{p_{s}\left(h_{j m}\right): j=1, \ldots, N\right\} \leq\|q\| / m$. Hence, if $m$ is large and $q_{m}$ is small enough, we get the required result.

Lemma 16. For each $b>0$ there is $m_{0} \in \mathbb{N}$ such that for any $m \geq m_{0}$ there is $q_{m}>0$ such that $\left\|\widetilde{A}_{m}(q)-\widehat{A}_{m}(q)\right\| \leq b\|q\|$ whenever $\|q\| \leq q_{m}$.

Proof. As in Lemma 15, by Lemma 13 it is enough to prove the inequality

$$
\sup _{\zeta \in \nu E}\left|e^{-\|q\| / m} \zeta-\zeta\right| \leq b\|q\|
$$

for small $\|q\|$. But for small $\|q\| / m$ we have $\left|1-e^{-\|q\| / m}\right| \leq 2\|q\| / m$. Hence, we get the required result. $\mathbb{R}^{N}$, if

Lemma 17 (cf. [Pol], Lemma 8). For any continuous mapping $F: \mathbb{R}_{+}^{2 N} \rightarrow$

$$
\|F(x)-A(x)\| \leq b\|x\| \quad \text { for } x \in B(0, r) \cap \mathbb{R}_{+}^{2 N},
$$

where $b=1 /(2 \sqrt{N})$, then there exists $q \in B(0, r) \cap \mathbb{R}_{+}^{2 N} \backslash\{0\}$ such that $F(q)=0$.

Proof. Define

$$
\mathcal{Q}:=\left\{\left(x_{1}, \ldots, x_{N}\right): 0<x_{j}<t_{0}, j=1, \ldots, N\right\}
$$

and

$$
\pi: \mathbb{R}^{N} \ni\left(x_{1}, \ldots, x_{N}\right) \rightarrow\left(x_{1}, t_{0}-x_{1}, \ldots, x_{N}, t_{0}-x_{N}\right) \in \mathbb{R}^{2 N},
$$

where $t_{0}=(2 \sqrt{N})^{-1} \min \{1, r\}$. It is easy to check that $\|\pi(l)\| \leq t_{0} \sqrt{N}$ for $l \in \overline{\mathcal{Q}}$ and $\pi(\mathcal{Q}) \subset B(0, r) \cap \mathbb{R}_{+}^{2 N}$. Note that

$$
\|F \circ \pi(l)-A \circ \pi(l)\| \leq b\|\pi(l)\| \leq t_{0} / 2 \quad \text { for } l \in \overline{\mathcal{Q}} .
$$

Consider the homotopy defined by the formula $\widetilde{F}_{t}=t F \circ \pi+(1-t) A \circ \pi$. It is enough to show that $0 \notin \widetilde{F}_{t}(\partial \mathcal{Q})$. Then from the homotopical invariance of the degree of mappings [Zei] we have $\operatorname{deg}(F \circ \pi, \mathcal{Q}, 0)=\operatorname{deg}(A \circ \pi, \mathcal{Q}, 0) \neq 0$, hence $0 \in F \circ \pi(\mathcal{Q})$.

It is easy to see that for any $l \in \partial \mathcal{Q}$,

$$
t_{0} \leq\|A \circ \pi(l)\| \leq\left\|\widetilde{F}_{t}(l)\right\|+t\|F \circ \pi(l)-A \circ \pi(l)\| \leq\left\|\widetilde{F}_{t}(l)\right\|+t_{0} / 2 .
$$

Hence, we get the required result.

Let us return to the proof of Lemma 9. By Lemmas 14-16 it follows that $\widehat{A}_{m}$ is continuous in $\mathbb{R}_{+}^{2 N}$ and for each $b>0$ there are $m \in \mathbb{N}$ and $q_{m}>0$ 
such that $\left\|\widehat{A}_{m}(q)-A(q)\right\| \leq b\|q\|$ for $\|q\| \leq q_{m}$. By Lemma 17 , for some $m$ we can find $q_{0}$ which is a solution of the equation $\widehat{A}_{m}\left(q_{0}\right)=0$. Hence, we have

$$
\Phi_{j}\left(\widehat{f}_{q_{0} m}\right)=a_{j} \quad \text { for } j=1, \ldots, N .
$$

But this contradicts the extremality of $f$, since $\widehat{f}_{q_{0} m}(E) \Subset D$.

3. Proof of Theorem 4. Before we prove the theorem we recall some auxiliary results.

Lemma 18. Let $\varphi \in H^{1}(E)$ be such that

$$
\frac{\varphi^{*}(\zeta)}{\prod_{k=1}^{m}\left(\zeta-\sigma_{k}\right)} \in \mathbb{R}_{>0} \quad \text { for a.a. } \zeta \in \partial E,
$$

where $\sigma_{k} \in \mathbb{C}, k=1, \ldots, m$. Then there exist $r \in \mathbb{R}$ and $\alpha_{k} \in \bar{E}, k=$ $1, \ldots, m$, such that

$$
\varphi(\zeta)=r \frac{\prod_{k=1}^{m}\left(\zeta-\alpha_{k}\right)\left(1-\bar{\alpha}_{k} \zeta\right)}{\prod_{k=1}^{m}\left(1-\bar{\sigma}_{k} \zeta\right)}, \quad \zeta \in E .
$$

This lemma is a generalization of Lemma 8.4.6 of [Jar-Pfl].

Proof. Put $\widetilde{\varphi}(\zeta)=\varphi(\zeta) \prod_{k=1}^{m}\left(1-\bar{\sigma}_{k} \zeta\right)$. Then $\widetilde{\varphi} \in H^{1}(E)$ and

$$
\frac{1}{\zeta^{m}} \widetilde{\varphi}^{*}(\zeta) \in \mathbb{R}_{>0} \quad \text { for a.a. } \zeta \in \partial E .
$$

Hence, it is enough to prove the lemma for $\sigma_{k}=0, k=1, \ldots, m$. Set

$$
P(\zeta)=\sum_{k=0}^{m} \frac{\varphi^{(k)}(0)}{k !} \zeta^{k}+\sum_{k=0}^{m-1} \frac{\overline{\varphi^{(k)}(0)}}{k !} \zeta^{2 m-k} .
$$

It is easy to see that if $\psi(\zeta):=(\varphi(\zeta)-P(\zeta)) / \zeta^{m}$, then $\psi \in H^{1}(E)$ and $\psi^{*}(\zeta) \in \mathbb{R}$ for a.a. $\zeta \in \partial E$. Hence $\psi \equiv 0$.

Let $t(\theta):=P\left(e^{i \theta}\right) / e^{i \theta m}$. We know that $t$ is $\mathbb{R}$-analytic and $t(\theta) \geq 0$ for $\theta \in \mathbb{R}$. If for some $\theta_{0} \in \mathbb{R}$ we have $t\left(\theta_{0}\right)=0$ then $t(\theta)=\left(\theta-\theta_{0}\right)^{k} \widetilde{t}(\theta)$, where $k$ is even.

Note that $\overline{P(1 / \bar{\zeta})}=P(\zeta) / \zeta^{2 m}$ and if $P(0)=0$, then $P(\zeta)=\zeta^{k} \widetilde{P}(\zeta)$, $\widetilde{P}(0) \neq 0, \operatorname{deg} \widetilde{P}=2 m-2 k$, and $\overline{\widetilde{P}(1 / \bar{\zeta})}=\widetilde{P}(\zeta) / \zeta^{2(m-k)}$. Now, it is enough to note that if $P\left(\zeta_{0}\right)=0, \zeta_{0} \neq 0$, then $P\left(1 / \bar{\zeta}_{0}\right)=0$ and if

$$
Q(\zeta):=\frac{P(\zeta)}{\left(\zeta-\zeta_{0}\right)\left(1-\bar{\zeta}_{0} \zeta\right)},
$$

then $\overline{Q(1 / \bar{\zeta})}=Q(\zeta) / \zeta^{2(m-1)}$.

Lemma 19. Let $S_{1}, S_{2}$ be singular inner functions and let $S_{1} S_{2} \equiv 1$. Then $S_{1}, S_{2} \equiv 1$. 
Proof. Suppose that

$$
S_{j}(z)=\exp \left(-\int_{0}^{2 \pi} \frac{e^{i t}+z}{e^{i t}-z} d \mu_{j}(t)\right), \quad j=1,2,
$$

where $\mu_{1}$ and $\mu_{2}$ are non-negative Borel measures, singular w.r.t. Lebesgue measure. Then $S_{1} S_{2} \equiv 1$ is equivalent to $\mu_{1}+\mu_{2}=0$. Since $\mu_{j} \geq 0, j=1,2$, we get $\mu_{1}=\mu_{2}=0$.

Proof of Theorem 4 . We know that $\varphi_{j}=B_{j} S_{j} F_{j}$, where $B_{j}$ is a Blaschke product, $S_{j}$ is a singular inner function and $F_{j}$ is an outer function. Take $s:=\left(F_{1}, \ldots, F_{n}\right)$. Note that $\left|\varphi_{j}^{*}(\zeta) / F_{j}^{*}(\zeta)\right|=1$ for a.a. $\zeta \in \partial E$ and

$$
\frac{\partial u}{\partial z_{j}}(\varphi)=p_{j} \frac{\left|\varphi_{j}\right|^{2 p_{j}}}{\varphi_{j}} \quad \text { for } j=1, \ldots, n .
$$

We want to show that the assumptions of Theorem 1 are satisfied. Let $u(z):=\sum_{j=1}^{n}\left|z_{j}\right|^{2 p_{j}}-1$ be the defining function for $\mathcal{E}(p)$.

We know that $\varphi_{j} \not \equiv 0, j=1, \ldots, n$. Hence $\nabla u\left(\varphi^{*}(\zeta)\right)$ exists for a.a. $\zeta \in \partial E$. We have

$$
\begin{aligned}
& \frac{\left|\varphi_{j}+F_{j} h_{j}\right|^{2 p_{j}}-\left|\varphi_{j}\right|^{2 p_{j}}-2 \operatorname{Re}\left(p_{j} \frac{\left|\varphi_{j}\right|^{2 p_{j}}}{\varphi_{j}} F_{j} h_{j}\right)}{\left|h_{j}\right|} \\
& =\left|\varphi_{j}\right|^{2 p_{j}} \frac{\left|1+\frac{F_{j}}{\varphi_{j}} h_{j}\right|^{2 p_{j}}-1-2 p_{j} \operatorname{Re}\left(\frac{F_{j}}{\varphi_{j}} h_{j}\right)}{\left|h_{j} \frac{F_{j}}{\varphi_{j}}\right|} .
\end{aligned}
$$

From the equality

$$
\lim _{z \rightarrow 0} \frac{|1+z|^{\alpha}-1-\alpha \operatorname{Re} z}{|z|}=0, \quad \alpha>0,
$$

we see that all the assumptions of Theorem 1 are satisfied.

Hence, by Corollary 3 , there exist $g \in H^{\infty}\left(E, \mathbb{C}^{n}\right)$ and $\varrho \in L^{\infty}(\partial E)$, $\varrho>0$, such that

$$
Q(\zeta) \varrho(\zeta) F_{j}^{*}(\zeta) \frac{\left|\varphi_{j}^{*}(\zeta)\right|^{2 p_{j}}}{\varphi_{j}^{*}(\zeta)}=g_{j}^{*}(\zeta) \quad \text { for a.a. } \zeta \in \partial E, j=1, \ldots, n,
$$

where $Q(\zeta)=\prod_{k=1}^{m}\left(\zeta-\sigma_{k}\right)$ is a polynomial witnessing the $m$-type. This is equivalent to

$$
Q(\zeta) \varrho(\zeta)\left|F_{j}^{*}(\zeta)\right|^{2 p_{j}}=B_{j}^{*}(\zeta) S_{j}^{*}(\zeta) g_{j}^{*}(\zeta) \quad \text { for a.a. } \zeta \in \partial E, j=1, \ldots, n \text {. }
$$

By Lemma 18 there exist $r_{j}>0$ and $\alpha_{k j} \in \bar{E}$ such that

$$
B_{j}^{*}(\zeta) S_{j}^{*}(\zeta) g_{j}^{*}(\zeta)=r_{j} \frac{\prod_{k=1}^{m}\left(\zeta-\alpha_{k j}\right)\left(1-\bar{\alpha}_{k j} \zeta\right)}{\prod_{k=1}^{m}\left(1-\bar{\sigma}_{k} \zeta\right)}
$$


and there exist $r_{0}>0$ and $\alpha_{k 0} \in \bar{E}$ such that

(4) $Q(\zeta) \varrho(\zeta)=\sum_{j=1}^{n} B_{j}^{*}(\zeta) S_{j}^{*}(\zeta) g_{j}^{*}(\zeta)=r_{0} \frac{\prod_{k=1}^{m}\left(\zeta-\alpha_{k 0}\right)\left(1-\bar{\alpha}_{k 0} \zeta\right)}{\prod_{k=1}^{m}\left(1-\bar{\sigma}_{k} \zeta\right)}$.

We have

$$
r_{0} \prod_{k=1}^{m}\left(\zeta-\alpha_{k 0}\right)\left(1-\bar{\alpha}_{k 0} \zeta\right)\left|F_{j}(\zeta)\right|^{2 p_{j}}=r_{j} \prod_{k=1}^{m}\left(\zeta-\alpha_{k j}\right)\left(1-\bar{\alpha}_{k j} \zeta\right) .
$$

Hence

$$
F_{j}(\zeta)=a_{j} \prod_{k=1}^{m}\left(\frac{1-\bar{\alpha}_{k j} \zeta}{1-\bar{\alpha}_{k 0} \zeta}\right)^{1 / p_{j}}
$$

where $a_{j} \in \mathbb{C} \backslash\{0\}$. From (6) it follows that

$$
B_{j}(\zeta)=\prod_{k=1}^{m}\left(\frac{\zeta-\alpha_{k j}}{1-\bar{\alpha}_{k j} \zeta}\right)^{r_{k j}}, \quad \text { where } r_{k j} \in\{0,1\} .
$$

Hence

$$
S_{j}(\zeta) g_{j}(\zeta)=r_{j} \frac{\prod_{k=1}^{m}\left(\zeta-\alpha_{k j}\right)^{1-r_{k j}}\left(1-\bar{\alpha}_{k j} \zeta\right)^{1+r_{k j}}}{\prod_{k=1}^{m}\left(1-\bar{\sigma}_{k} \zeta\right)} .
$$

Since the right-hand side is an outer function, from Lemma 19 we conclude that $S_{j} \equiv 1, j=1, \ldots, n$.

From (5) and (6) we see that $\left|a_{j}\right|^{2 p_{j}}=r_{j} / r_{0}$ and from (3) and (4) it follows that

$$
\sum_{j=1}^{n}\left|a_{j}\right|^{2 p_{j}} \prod_{k=1}^{m}\left(\zeta-\alpha_{k j}\right)\left(1-\bar{\alpha}_{k j} \zeta\right)=\prod_{k=1}^{m}\left(\zeta-\alpha_{k 0}\right)\left(1-\bar{\alpha}_{k 0} \zeta\right), \quad \zeta \in E .
$$

So, we get the required result.

\section{The case of complex geodesics}

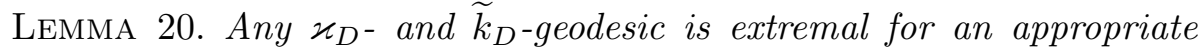
problem $(\mathcal{P})$ of 1-type.

Proof. The case of a $\varkappa_{D}$-geodesic. Consider problem $(\mathcal{P})$ with linear functionals such that:

- $N=4 n$,

- $w_{j}:=(0, \ldots, 1, \ldots, 0)$ and $a_{j}:=\operatorname{Re} z_{j}$ for $j=1, \ldots, n$,

- $w_{j}:=(0, \ldots,-i, \ldots, 0)$ and $a_{j}:=\operatorname{Im} z_{j}$ for $j=n+1, \ldots, 2 n$,

- $w_{j}:=(0, \ldots, 1 / \zeta, \ldots, 0)$ and $a_{j}:=\operatorname{Re} X_{j}$ for $j=2 n+1, \ldots, 3 n$,

- $w_{j}:=(0, \ldots,-i / \zeta, \ldots, 0)$ and $a_{j}:=\operatorname{Im} X_{j}$ for $j=3 n+1, \ldots, 4 n$,

where $z \in D$ and $X \in \mathbb{C}^{n} \backslash\{0\}$. 
It is easy to see that the corresponding linear functionals are linearly independent and problem $(\mathcal{P})$ is of 1-type.

Let us show that any $\varkappa_{D}$-geodesic $f$ for $(z, X)$ is extremal for this problem $(\mathcal{P})$. Suppose that there exists a mapping $g \in \mathcal{O}(E, D)$ such that $g(0)=z, g^{\prime}(0)=X$, and $g(E) \Subset D$. Write $\widetilde{g}(\zeta):=g(\zeta)+\zeta t X$, where $t>0$ will be defined later. Then $\widetilde{g}(0)=g(0)=z$ and $\widetilde{g}^{\prime}(0)=g^{\prime}(0)+t X=(1+t) X$. If we take $t$ such that $\widetilde{g}(E) \subset D$ (that is possible, because $g(E) \Subset D)$, then we have a contradiction with $f$ being a $\varkappa_{D}$-geodesic.

The case of a $\widetilde{k}_{D}$-geodesic. Consider problem $(\mathcal{P})$ with linear functionals such that $f \in \mathcal{O}(E, D)$ is extremal iff $f(0)=z, f(\sigma)=w$, where $\sigma>0$, and there is no mapping $g \in \mathcal{O}(E, D)$ such that

(1) $g(0)=z, g(\sigma)=w$,

(2) $g(E) \Subset D$.

(The functions $w_{j}$ in this case can be constructed similarly to the case of a $\varkappa_{D}$-geodesic. It is enough to replace $1 / \zeta$ by $1 /(\zeta-\sigma)$ and $-i / \zeta$ by $-i /(\zeta-\sigma)$.) It is easy to see that the relevant linear functionals are linearly independent and that the problem $(\mathcal{P})$ is of 1-type.

Let us show that any $\widetilde{k}_{D}$-geodesic $f$ is extremal for this problem. Suppose that there exists a mapping $g \in \mathcal{O}(E, D)$ such that $g(0)=z, g(\sigma)=w$, and $g(E) \Subset D$. Define

$$
\widetilde{g}(\zeta):=g(\zeta)+\frac{\zeta}{t \sigma}(g(\sigma)-g(t \sigma))
$$

where $0<t<1$ will be defined later. Then $\widetilde{g}(0)=g(0)=z$ and $\widetilde{g}(t \sigma)=$ $g(\sigma)=w$. If we take $t$ such that $\widetilde{g}(E) \subset D$ (use $g(E) \Subset D)$, then we have a contradiction, because $f$ is a $\widetilde{k}_{D}$-geodesic.

Acknowledgements. The ideas of the paper come from fruitful conversations with Professors M. Jarnicki and W. Zwonek. I would like to thank them.

\section{References}

[Hay-Ken] W. K. Hayman and P. B. Kennedy, Subharmonic Functions, Vol. I, Academic Press, 1976.

[Jar-Pfl] M. Jarnicki and P. Pflug, Invariant Distances and Metrics in Complex Analysis, Walter de Gruyter, 1993.

[Jar-Pfl-Zei] M. Jarnicki, P. Pflug, and R. Zeinstra, Geodesics for convex complex ellipsoids, Ann. Scuola Norm. Sup. Pisa 20 (1993), 535-543.

[Pan] M.-Y. Pang, Smoothness of the Kobayashi metric of non-convex domains, Internat. J. Math. 4 (1993), 953-987.

[Pfl-Zwo] P. Pflug and W. Zwonek, The Kobayashi metric for non-convex complex ellipsoids, preprint, 1994. 
[Pol] E. A. Poletskiǔ, The Euler-Lagrange equations for extremal holomorphic mappings of the unit disk, Michigan Math. J. 30 (1983), 317-333.

[Zei] E. Zeidler, Nonlinear Functional Analysis and its Applications, Vol. I: Fixed-Point Theorems, Springer, 1986.

INSTITUTE OF MATHEMATICS

JAGIELLONIAN UNIVERSITY

REYMONTA 4

30-059 KRAKÓW, POLAND

E-mail: EDIGARIA@IM.UJ.EDU.PL

Reçu par la Rédaction le 9.1.1995 